\title{
O Teste Gestáltico Visomotor de Bender na avaliação clínica de Crianças ${ }^{1}$
}

\author{
Roselaine Berenice Ferreira ${ }^{2}$ - Universidade de Santa Cruz do Sul, Santa Cruz do Sul, Brasil \\ Cristiane Friedrich Feil - Pontificia Universidade do Rio Grande do Sul, Porto Alegre, Brasil \\ Maria Lucia Tiellet Nunes - Pontificia Universidade do Rio Grande do Sul, Porto Alegre, Brasil
}

\begin{abstract}
Resumo
Este estudo avaliou os erros mais freqüentes cometidos por crianças no Bender, pelo método Koppitz, e identificou sua relação com as variáveis idade e sexo. Analisou-se 1111 protocolos de avaliação psicológica, contemplando meninos e meninas, com idades entre cinco e doze anos, os quais apresentavam dificuldades emocionais, cognitivas e neurológicas, compondo uma amostra clínica. Constatou-se que os erros mais comuns frente à cópia do Bender foram a Distorção da Forma nas figuras 7 e 8 e a Rotação na figura 7. Através do teste do Qui-quadrado identificou-se relação significativa entre erros nas figuras $A, 1,2,4,6,7$ e 8 e a variável idade $(p=0,000)$. A variável sexo apresentou correlação significativa somente em erros nas figuras 4 e 7 no item rotação $(\mathrm{p}<0,005)$.

Palavras-chave: teste Bender; testes psicológicos; maturidade.
\end{abstract}

\section{Bender Visual-motor Gestalt Test in the Children's Clinical Assessment}

\begin{abstract}
This study discusses the most frequent errors committed by children in the Bender Test, assessed by the Koppitz method, and identified the correlation regarding age and sex. 1111 psychological assessment protocols were examined, including boys and girls, aged five and twelve years, which had emotional, cognitive and neurological difficulties, composing a clinical sample. The most frequent errors in the Bender test were distortion of form in Figures 7 and 8, and Rotation in Figure 7. Using Qui-square test was identified a significant relationship between errors in Figures $A, 1,2,4,6,7$ and 8 and variable age $(p=0000)$ was found. The variable sex showed significant correlation only in error of Figures 4 and 7 on the rotation item $(\mathrm{p}<0005)$.

Keywords: Bender test; psychological test; maturity.
\end{abstract}

\section{Introdução}

A avaliação psicológica clínica é processo que permite descrever e compreender a personalidade de um indivíduo, investigar sintomas, possibilitando estimar o prognóstico do caso e a estratégia e/ou abordagem terapêutica. É um processo científico, de tempo limitado que ao lançar mão de técnicas e testes psicológicos busca entender problemas à luz de pressupostos teóricos para identificar e avaliar aspectos específicos do caso e prever seu curso possível, comunicando os resultados aos sujeitos envolvidos (Arzeno, 1995; Cunha, 2000; Ocampo, Arzeno \& Piccolo, 1981).

$\mathrm{Na}$ visão de Wechsler e Guzzo (2005), o uso dos instrumentos psicológicos proporciona dados importantes e a avaliação fornece significado aos resultados, considerando o contexto em que o examinando está inserido. Nesse sentido, verifica-se a importância da utilização de instrumentos psicológicos na avaliação psicológica infantil. Atualmente, a disponibilidade de instrumentos que possuam estudos de normas para a realidade brasileira ainda é escassa, determinando a urgência de pesquisas nessa direção.

Kaefer (2006) destaca que, no presente momento, em avaliação psicológica, os instrumentos mais utilizados pelos psicólogos são: Escala Wechsler de Inteligência para Crianças (WISC-III), Teste Gestáltico Visomotor Bender, Desenho da Figura Humana, Técnica de Rorschach e Teste das Fábulas.

\section{Proposta maturacional do Bender}

O Teste Gestáltico Visomotor de Bender foi construído por Lauretta Bender, em 1938, inspirado nas figuras de Wertheimer de 1923, que estudou a percepção visual. No decorrer de seus estudos, Bender (1955) propôs a formatação do instrumento para um total de nove cartões, cada um deles medindo $14,9 \mathrm{~cm}$ de comprimento por $10,1 \mathrm{~cm}$ de altura.

Consiste de cartelas em cor branca, compostas por figuras diferenciadas que se encontram desenhadas em cor preta. São estímulos formados por linhas contínuas ou pontos, curvas sinuosas ou ângulos.

A partir do estudo da própria autora (Bender, 1955), é possível identificar que todas as figuras do

\footnotetext{
${ }^{1}$ Este estudo deriva da tese de doutorado da primeira autora, bolsista CAPES de 2004 a 2007, orientada pela terceira autora; a segunda autora é bolsista de Iniciação Científica pela FAPERGS.

2 Endereço para correspondência:

Av. Independência, 2293 - 96825-900 - B. Universitário, Santa Cruz do Sul-RS, Brasil

Fone: (51) 3717-7388

E-mail: mrsilva@unisc.br
} 
instrumento são regidas por três princípios básicos da Gestalt: Princípio do Fechamento, da Proximidade e da Continuidade. Sendo assim, cinco figuras do teste são formadas por linhas retas ou contínuas (figuras A, 4, 6, 7 e 8 ) e quatro figuras, por pontos ou círculos (figuras 1, 2, 3 e 5).

Um dos sistemas de correção do Bender para crianças, muito enfatizado na literatura, é o de Elisabeth Koppitz (1960/1989). Em seus estudos iniciais para a normatização da Escala de Maturação, essa autora utilizou uma amostra de 1.104 crianças provenientes de escolas públicas dos Estados Unidos, todas com idades de 5 anos a 10 anos e 11 meses. O método de correção utilizado pela autora considera 30 itens pontuados nas 9 figuras apresentadas, sendo esses itens condensados em quatro tipos: distorção da forma, rotação, integração e perseveração.

O sistema Koppitz é o mais conhecido aqui no Brasil, sendo um sistema que se propõe a avaliar a maturidade visomotora. Nesse propósito, Kacero (2005) salienta que o ato visomotor é extremamente complexo e maturacional.

Bender (1955) relata o estudo de Bernstein que elaborou um quadro de normas quanto à maturação visomotora em crianças quantificando, de acordo com a idade, a forma com que elas desenhavam as figuras do Bender. Para isso, analisou o estudo de Lauretta Bender composto de 800 crianças. Identificou que $90 \%$ das crianças de quatro anos executaram a Figura A em formato de dois círculos; aos cinco anos, 85\% delas já executavam o quadrado, mas na posição horizontal (e não inclinado como é o estímulo).

Somente aos seis anos, a Figura A estava sendo desenhada conforme o estímulo apresentado, mas com tendência de haver dificuldade de integração de uma subparte em outra: círculo e quadrado bem feitos, mas separados entre si. Aos sete anos, a figura era copiada sem erros, demonstrando a aquisição da integridade visomotora nessa faixa etária: fase da escolarização. O maior grau de dificuldade ocorreu na reprodução das figuras 3 e 7, pois somente aos 10 anos eram copiadas praticamente sem erros, o que confirmou a observação já feita por Lauretta Bender.

Verificou, também, que as crianças de 6 anos já possuíam a capacidade de copiar ângulos, mas sem nenhuma orientação espacial, pois a tendência é executar a cópia não prestando atenção à posição da figura, que, no caso da Figura 7, está posicionada de forma transversal. A Figura 8, todavia, não evidencia tanta dificuldade como a Figura 7 , por possuir o desenho disposto de forma horizontal, sob ponto de vista da percepção espacial. Já aos 7 anos, a criança conseguia reproduzi-la, praticamente, sem erros.

Bender (1955) salienta, ainda, que a criança em idade escolar (a partir dos seis anos) possui a capacidade de ler e escrever, já que suas funções visomotoras estão mais amadurecidas, ou seja, similares às funções de um adulto. Contudo, é a partir dos 12 anos que essa capacidade se torna consolidada.

\section{Objetivos}

Este estudo tem por objetivo identificar a frequência dos tipos de erro, e a relação de sexo e idade no desempenho quanto ao Bender.

\section{Método}

Trata-se de uma pesquisa documental, via protocolos de arquivo de avaliações psicológicas, cedido por profissional que trabalha com avaliação psicológica. Foram analisados 1.111 protocolos de avaliação psicológica de crianças entre cinco e doze anos de idade, escolhidos por conveniência e divididos em oito grupos de acordo com a faixa etária, sendo o intervalo de um ano em cada grupo, e em dois grupos, de acordo com o sexo.

$\mathrm{O}$ arquivo de protocolos pertencia a uma profissional que cedeu seu uso para fins desta pesquisa. Tais protocolos compreenderam o período entre 2000 e 2007, sendo compostos de resultados de crianças de diversas cidades do estado do Rio Grande do Sul, contendo, dentre outros, os resultados do Teste Gestáltico Visomotor de Bender.

Como se trata de uma pesquisa documental, via protocolos de arquivo de avaliações psicológicas, foram selecionados, por conveniência, casos que continham o Bender. As informações extraídas foram transportadas para o banco de dados no programa estatístico SPSS versão 11.5 for Windows, sem a identificação nominal de cada indivíduo, como forma de garantir o anonimato do grupo avaliado.

Para a avaliação das figuras do Bender foi utilizado o método Koppitz (1989), em que se avaliam os erros em quatro categorias: distorção da forma, integração, rotação e perseveração. Cada erro equivale a 1 ponto, portanto um desempenho ruim pressupõe um número maior de pontos. $\mathrm{O}$ escore final, que pelo sistema de correção Koppitz é feito de forma numérica, sendo possível um escore de zero a trinta pontos, foi neste estudo transformado em categoria nominal: "abaixo", "compatível" e "acima" para a idade da criança. Os dados foram analisados por estatística descritiva (frequências, médias, desvio padrão e teste do Qui-quadrado) e as informações extraídas dos protocolos foram 
transportadas para o banco de dados no programa estatístico SPSS versão 11.5 for Windows.

Como se trata de pesquisa de arquivo, a psicóloga em cuja responsabilidade reside a guarda do material cedeu acesso ao seu uso para as autoras, que mantêm, por sua vez, o mesmo compromisso ético de guardar sigilo da identidade das crianças. Esta foi ainda assegurada pelo fato de que cada criança ficou registrada de acordo com um número, sendo, então, seu código de identificação.

\section{Resultados e discussão}

Os resultados encontrados apontam para a constatação de que o Bender é um instrumento que se mostra sensível à identificação de problemas de ordem neurológica, em nível maturacional. A amostra consistiu de 1111 crianças clínicas, encaminhadas para avaliação psicológica, apresentando, dessa forma, problemas clínicos dos mais diversos.

Serão apresentados, inicialmente, resultados em relação à idade, na busca de associações entre essa variável e os erros mais freqüentes no Bender. Num segundo momento, o objetivo é examinar a associação entre sexo e os erros mais freqüentes.

\section{Estudo sobre Idade e Erros no Bender}

Trata-se de examinar a classificação dos escores obtidos no Bender em termos de a criança ter uma produção compatível, abaixo ou acima do esperado para sua idade.

A Tabela 1 apresenta a frequência dos resultados no Bender, categorizados em abaixo, compatível e acima, em relação à idade. É possível observar que as crianças demonstraram dificuldades na execução do teste: $73,4 \%$ da amostra se encontra na categoria abaixo da idade cronológica. As crianças de 12 anos apresentaram maior percentual de resultados abaixo de sua idade cronológica $(98,2 \%)$, o que se compreende em se tratando de amostra clínica, pois tais resultados condizem com as dificuldades expressadas por essa amostra.

Ao mesmo tempo, em aparente paradoxo, crianças com cinco anos evidenciaram produção mais compatível para a idade $(65,1 \%)$. Entretanto, como o teste tem base maturacional, o levantamento de escores permite que os erros sejam aceitos por serem compatíveis com o desenvolvimento neurológico esperado para a idade.

A idade de 7 anos, assim como as idades de $9 \mathrm{e}$ 12 anos, apresentou desempenho abaixo do esperado para a idade, consequentemente, aumentando o número de erros no teste (respectivamente, 64,9\%, $84,9 \%$ e $98,2 \%$ ). Além do próprio sintoma que levou a criança à avaliação psicológica, é possível inferir que essas idades representam momentos desenvolvimentais importantes, como o início da alfabetização e o ingresso na puberdade, respectivamente, em consequência para o desempenho no Bender.

A literatura aponta para a constatação de que o ingresso da criança na fase escolar representa um momento importante em seu desenvolvimento. É uma fase de grandes transformações nas áreas cognitiva, emocional e social, processo semelhante ao do período em que inicia seu processo adolescente.

Tabela 1 - Distribuição de Frequência de resultados por idade

\begin{tabular}{lccccc}
\hline \multicolumn{1}{c}{ Idade } & $\mathrm{N}$ & Abaixo & Compativel & Acima & Total \\
\hline 5 anos & 43 & $30,2 \%$ & $65,1 \%$ & $4,7 \%$ & $100,0 \%$ \\
6 anos & 109 & $56,0 \%$ & $35,8 \%$ & $8,3 \%$ & $100,0 \%$ \\
7 anos & 186 & $69,4 \%$ & $29,5 \%$ & $2,2 \%$ & $100,0 \%$ \\
8 anos & 220 & $64,5 \%$ & $30,0 \%$ & $5,5 \%$ & $100,0 \%$ \\
9 anos & 186 & $84,9 \%$ & $11,3 \%$ & $3,8 \%$ & $100,0 \%$ \\
10 anos & 190 & $81,1 \%$ & $17,4 \%$ & $1,6 \%$ & $100,0 \%$ \\
11 anos & 122 & $85,2 \%$ & $13,9 \%$ & $0,8 \%$ & $100,0 \%$ \\
12 anos & 55 & $98,2 \%$ & $1,8 \%$ & $0,0 \%$ & $100,0 \%$ \\
\hline Total & 1111 & $73,4 \%$ & $23,2 \%$ & $3,5 \%$ & $100,0 \%$ \\
\hline
\end{tabular}


Tabela 2 - Distribuição de frequência de erros de distorção da forma, integração, rotação e perseveração nas Figuras.

\begin{tabular}{lcccccc}
\hline \multicolumn{1}{c}{ Figuras } & $\mathrm{DF}(a)$ & $\mathrm{DF}(b)$ & $\operatorname{Int}(a)$ & $\operatorname{Int}(b)$ & Rot & Pers \\
\hline Figura A & $18,6 \%$ & $18,9 \%$ & $27,7 \%$ & - & $18,6 \%$ & - \\
Figura 1 & $23,9 \%$ & - & --- & - & $9,3 \%$ & $14,7 \%$ \\
Figura 2 & - & - & $9,3 \%$ & - & $30,0 \%$ & $7,5 \%$ \\
Figura 3 & $39,2 \%$ & - & $32,9 \%$ & $5,7 \%$ & $31,8 \%$ & - \\
Figura 4 & - & - & $38,0 \%$ & - & $52,3 \%$ & - \\
Figura 5 & $39,0 \%$ & - & $8,1 \%$ & $10,4 \%$ & $23,4 \%$ & - \\
Figura 6 & $55,2 \%$ & $8,4 \%$ & $14,7 \%$ & - & - & $30,6 \%$ \\
Figura 7 & $37,9 \%$ & $84,2 \%$ & $38,3 \%$ & - & $74,2 \%$ & - \\
Figura 8 & $77,8 \%$ & - & - & - & $11,0 \%$ & - \\
\hline
\end{tabular}

DF(a): Distorção da Forma, item a; DF(b): Distorção da Forma, item b; Int(a): Integração, item a; Int(b): Integração, item b; Rot: Rotação; Pers: Perseveração; (-) Item inexistente na figura.

Outra forma de examinar os erros cometidos pelas crianças em relação ao teste é verificar a frequência de erros no decorrer da execução da cópia do total das nove figuras do Bender. Também foi possível constatar que o item distorção da forma teve maiores percentuais de erro na Figura 7 - item b $(84,2 \%)$ e na Figura $8(77,8 \%)$, conforme a Tabela 2.

Outro item que revelou alto índice de erros foi o item rotação na Figura 7 (74,2\%). Os erros nos itens integração e perseveração não foram tão comuns. Sisto, Noronha e Santos (2004) verificaram que o item distorção da forma é mais sensível à análise que os demais itens de Koppitz.
Noronha e Mattos (2006) identificaram um percentual de erros de $77,7 \%$ nas Figuras 7 e 8 . Igualmente identificaram que o item rotação foi o menos pontuado, obtendo menor percentual na Figura $1(1,2 \%)$. O menor percentual de erros, na amostra clínica do presente estudo, ocorreu na Figura 3, no item integração(b), com 5,7\% de crianças no total. Em contrapartida, foi identificado um percentual de erros considerável no item rotação, na Figura 7 (74\%).

Em vista de as Figuras 7 e 8 evidenciarem maior dificuldade na execução da cópia, pela criança, a seguir será examinada a maior ocorrência de erros, levandose em conta a idade.

Tabela 3 - Frequência de acertos/erros na figura 7 (distorção da forma-b) por idade

\begin{tabular}{lcccccccc}
\hline \multicolumn{1}{c}{ Idade } & \multicolumn{9}{c}{ Figura 7 - Distorção da Forma(b) } & \multicolumn{2}{c}{ Não fez } & \multicolumn{2}{c}{ Total } \\
\cline { 2 - 10 } & $N$ & $\%$ & $N$ & $\%$ & $N$ & $\%$ & A & $\%$ \\
\hline 5 anos & 3 & 7,0 & 40 & 93,0 & 0 & 0,0 & 43 & 100,0 \\
6 anos & 9 & 8,3 & 100 & 91,7 & 0 & 0,0 & 109 & 100,0 \\
7 anos & 12 & 6,5 & 174 & 93,5 & 0 & 0,0 & 186 & 100,0 \\
8 anos & 21 & 9,5 & 198 & 90,0 & 1 & 0,5 & 220 & 100,0 \\
9 anos & 29 & 15,6 & 157 & 84,4 & 0 & 0,0 & 186 & 100,0 \\
10 anos & 45 & 23,7 & 145 & 76,3 & 0 & 0,0 & 190 & 100,0 \\
11 anos & 37 & 30,3 & 85 & 69,7 & 0 & 0,0 & 122 & 100,0 \\
12 anos & 19 & 34,5 & 36 & 65,5 & 0 & 0,0 & 55 & 100,0 \\
\hline Total & 175 & 15,8 & 935 & 84,2 & 1 & 0,1 & 1.111 & 100,0 \\
\hline
\end{tabular}

A Tabela 3 demonstra que na idade de 7 anos os erros na execução da cópia da Figura 7 são mais cometidos, totalizando uma frequência de $93,5 \%$ no item distorção da forma se comparado a outras idades. Entre 5 e 6 anos, o percentual de erro foi igualmente elevado $(93,0 \%$ e $91,7 \%$, respectivamente), mas, como destaca Koppitz (1989), tal erro nessas idades é compatível.
$\mathrm{Na}$ Tabela 4 constata-se um percentual maior de erros $(89,9 \%)$ na faixa etária de 6 anos para a Figura 7, item rotação. Koppitz (1989) identificou que até essa idade a criança possui a tendência de cometer esse tipo de erro nessa figura. Porém, nesta amostra clínica, também é identificado que, aos 7 e 8 anos, a criança apresenta um percentual de erros ainda elevado (83,3\% e 80,5\%, respectivamente). 
Tabela 4 - Frequência de acertos/erros na figura 7 (rotação) por idade

\begin{tabular}{lcccccccc}
\hline & \multicolumn{9}{c}{ Figura 7 - Rotação } \\
\hline \multicolumn{1}{c}{ Idade } & \multicolumn{2}{c}{ Acerto } & \multicolumn{2}{c}{ Erro } & \multicolumn{2}{c}{ Não fez } & \multicolumn{2}{c}{ Total } \\
& $\mathrm{N}$ & $\%$ & $\mathrm{~N}$ & $\%$ & $\mathrm{~N}$ & $\%$ & $\mathrm{~N}$ & $\%$ \\
\hline 5 anos & 7 & 16,3 & 36 & 83,7 & 0 & 0,0 & 43 & 100,0 \\
6 anos & 11 & 10,1 & 98 & 89,9 & 0 & 0,0 & 109 & 100,0 \\
7 anos & 31 & 16,7 & 155 & 83,3 & 0 & 0,0 & 186 & 100,0 \\
8 anos & 42 & 19,1 & 177 & 80,5 & 1 & 0,5 & 220 & 100,0 \\
9 anos & 53 & 28,5 & 133 & 71,5 & 0 & 0,0 & 186 & 100,0 \\
10 anos & 67 & 35,3 & 123 & 64,7 & 0 & 0,0 & 190 & 100,0 \\
11 anos & 50 & 41,0 & 72 & 59,0 & 0 & 0,0 & 122 & 100,0 \\
12 anos & 25 & 45,5 & 30 & 54,5 & 0 & 0,0 & 55 & 100,0 \\
\hline Total & 285 & 25,7 & 824 & 74,2 & 1 & 0,1 & 1.111 & 100,0 \\
\hline
\end{tabular}

Por sua vez, a Tabela 5 evidencia igualmente a frequência de erros cometidos, porém, na Figura 8. No item distorção da forma, o percentual de erro chegou a $88,7 \%$ para a idade de 7 anos. $\mathrm{Na}$ idade de 6 anos, o percentual foi elevado $(87,2 \%)$, ao passo que Koppitz (1989) também considera compatível.

Segundo Clawson (1992), figuras multiangulares como as 7 e 8 oferecem muita dificuldade para crianças pequenas pela dificuldade perceptual de fazer linhas oblíquas. Por esse motivo, a interpretação angular da Figura 7 não é confiável antes da idade mental de dez anos; já na Figura 8, antes da idade dos oito anos. Portanto, ela parece ter estabelecido um ponto de corte para o fator idade, sendo possível avaliar com mais confiabilidade essas figuras somente em crianças com idade mais avançada.

$\mathrm{Na}$ idade de 7 anos a criança é capaz de fazer muito bem linhas oblíquas nas figuras A e 8, unindo as subpartes dessas duas figuras melhor do que fazia aos seis anos (Clawson, 1992). A mesma autora ainda afirma que a figura mais difícil de ser desenhada é a Figura 3, com seus ângulos de amplitude crescente. A maioria das crianças de 11 anos consegue reproduzir satisfatoriamente esses ângulos. Neste sentido, constatam-se diferenças entre o estudo de Clawson e o presente estudo, em que se utilizou o método Koppitz. A Figura 3, não evidenciou grandes dificuldades para execução da cópia, além do que as crianças de 7 e 8 anos cometeram mais erros nas figuras 7 e 8 .

$\mathrm{Na}$ associação entre idade e erros cometidos, destaca-se que para a Figura 3, no item rotação, não foi encontrado resultado significativo $\left(\chi^{2}=7,655\right.$; $p=0,366)$, assim como para a Figura 5, item distorção da forma $\left(\chi^{2}=22,469 ; p=0,097\right)$. Para as demais figuras, a associação entre idade e erro foi significativa, com o valor de $p=0,000$.

Ohlweiler e Guardiola (2006) destacam a evolução da capacidade visual da criança, afirmando que consiste num desenvolvimento maturacional, iniciando com a percepção dos objetos. Reiteram que somente depois da idade de quatro anos a criança diferencia as formas euclidianas, distinguindo ângulos e dimensões. Entre quatro e cinco anos discrimina quadrado de retângulo, círculo de elipse. A partir de 8 e 9 anos desenha corretamente em perspectiva.

Tabela 5 - Frequência de acertos/erros na Figura 8 (distorção da forma) por idade

\begin{tabular}{|c|c|c|c|c|c|c|c|c|}
\hline \multicolumn{9}{|c|}{ Figura 8 - Distorção da Forma } \\
\hline \multirow[t]{2}{*}{ Idade } & \multicolumn{2}{|c|}{ Acerto } & \multicolumn{2}{|c|}{ Erro } & \multicolumn{2}{|c|}{ Não fez } & \multicolumn{2}{|c|}{ Total } \\
\hline & $\mathrm{N}$ & $\%$ & $\mathrm{~N}$ & $\%$ & $\mathrm{~N}$ & $\%$ & $\mathrm{~N}$ & $\%$ \\
\hline 5 anos & 6 & 14,0 & 37 & 86,0 & 0 & 0,0 & 43 & 100,0 \\
\hline 6 anos & 14 & 12,8 & 95 & 87,2 & 0 & 0,0 & 109 & 100,0 \\
\hline 7 anos & 21 & 11,3 & 165 & 88,7 & 0 & 0,0 & 186 & 100,0 \\
\hline 8 anos & 31 & 14,1 & 188 & 85,4 & 1 & 0,5 & 220 & 100,0 \\
\hline 9 anos & 46 & 24,7 & 140 & 75,3 & 0 & 0,0 & 186 & 100,0 \\
\hline 10 anos & 61 & 32,1 & 129 & 67,9 & 0 & 0,0 & 190 & 100,0 \\
\hline 11 anos & 45 & 36,9 & 77 & 63,1 & 0 & 0,0 & 122 & 100,0 \\
\hline 12 anos & 22 & 40,0 & 33 & 60,0 & 0 & 0,0 & 55 & 100,0 \\
\hline Total & 246 & 22,1 & 864 & 77,8 & 1 & 0,1 & 1.111 & 100,0 \\
\hline
\end{tabular}




\section{2 - Estudo sobre sexo e erros no Bender}

$\mathrm{Na}$ amostra pesquisada, foi estudada a associação entre sexo e os erros cometidos quando da cópia das figuras do Bender. Os escores obtidos

por meninos e meninas foram categorizados em termos de a criança ter uma produção compatível, abaixo ou acima do esperado para sua idade.

Tabela 6 - Distribuição de Freqüência dos Resultados do Bender por Sexo

\begin{tabular}{rcccc}
\hline Sexo & $N$ & Abaixo $(\%)$ & Compatível $(\%)$ & Acima $(\%)$ \\
\hline Masculino & 665 & 73,5 & 22,4 & 4,1 \\
Feminino & 446 & 73,1 & 24,4 & 2,5 \\
\hline Total & 1111 & 73,4 & 23,2 & 3,4 \\
\hline
\end{tabular}

$\mathrm{Na}$ Tabela 6 constata-se o número maior de meninos nos protocolos de avaliação psicológica, amostra deste estudo. Ao mesmo tempo, observa-se que não ocorreram diferenças na execução da cópia das figuras, pois tanto meninos como meninas evidenciaram percentual de escores abaixo para sua idade (73,5\% para meninos e $73,1 \%$ para meninas).

Não houve associação significativa entre sexo e os erros nas figuras do Bender $(p>0,05)$. Por sua vez, algumas figuras demonstraram significância como a Figura 4, no item rotação $\left(\chi^{2}=10,944\right.$; $p=0,004)$ e a Figura 7 , item rotação $\left(\chi^{2}=14,397\right.$; $p=0,001)$.

Em relação à variável sexo relacionada com os erros do Bender, segundo Oliveira (1985), também não foram encontradas diferenças significativas. Noronha, Santos e Sisto (2007) constataram que a variável sexo não possui associação significativa com a produção no Bender, comentando que não há evidências que meninos e meninas possuam diferenças no processo de amadurecimento em nível perceptomotor.

\section{Conclusão}

Conclui-se este estudo, atentando ao fato que esta foi uma amostra clínica, ou seja, foram utilizados arquivos de avaliações psicológicas de crianças na faixa etária de cinco a doze anos. Desta forma, foi identificado que os erros mais cometidos na execução do Bender foram os itens distorção da forma (b) na Figura 7, distorção da forma na Figura 8, além de rotação na Figura 7.

Além disso, na faixa etária de 7 anos, os itens distorção da forma (b) para a Figura 7 e distorção da forma para a Figura 8 apresentaram maior frequência de erros. Já nas faixas etárias de 5 e 6 anos, o item rotação evidenciou um percentual maior de erros cometidos, ao passo que, aos 7 e 8 anos, verifica-se que o percentual de erros ainda permanece elevado.
$\mathrm{Na}$ associação entre idade e tipo de erro constatou-se que o Bender possui associação significativa com a idade da criança, com exceção de dois itens de correção para duas figuras distintas, a saber: o item rotação, na Figura 3 e o item distorção da forma na Figura 5. Por sua vez, na associação entre sexo e tipo de erro foi encontrada associação significativa somente nas Figuras 4 e 7 no item rotação.

Essas constatações encontram respaldo na literatura, tendo Koppitz (1989) destaca o comportamento da criança tem relação ao Bender: crianças menores, com idades entre 5 e 7 anos, evidenciam maior dificuldade na cópia das figuras; em razão disso, os erros cometidos pelas crianças da amostra são mesmo mais comuns de ocorrerem. A partir da idade de 8 anos, pelo amadurecimento do sistema nervoso central, a criança já tem condições de executar a cópia de forma a não produzir tantos erros em nível de distorção da forma, rotação, integração e perseveração. Esses mesmos erros, quando muito frequentes em algumas figuras, evidenciam dificuldades maturacionais em nível neurológico na criança.

No presente estudo, a faixa etária de 7 anos evidenciou um percentual alto de erros nas Figuras 7 e 8. Após essa idade, percebeu-se que os erros iam diminuindo para essas figuras. Koppitz (1989), em seu estudo, identificou que os erros cometidos na Figura 7 possuem baixo valor diagnóstico, pois nenhuma criança foi capaz de desenhar corretamente a figura antes dos 8 anos. Já na Figura 8, crianças após 6 anos evidenciavam facilidade na cópia da mesma. O mesmo ocorreu na Figura 7, para o item rotação, para o qual Koppitz constatou que crianças até 6 anos possuem dificuldade em sua reprodução. Após essa idade, torna-se um tipo de erro com alto valor diagnóstico para dificuldades neurológicas ou maturacionais.

Sisto, Noronha e Santos (2004) investigaram o erro de distorção da forma nas figuras do Bender. 
Identificaram diferenças significativas em relação à idade da criança, ou seja, conforme aumenta a idade, diminui o erro de distorção. No presente estudo, foi constatado que, além do item distorção da forma ser significativo, o item rotação também o é.

Koppitz (1989) evidenciou, em seu estudo normativo, que a partir dos 10 anos a criança é capaz de copiar as figuras do Bender praticamente sem erros. Ao mesmo tempo, informou que nas idades de 5 a 7 anos as crianças têm mais propensão a cometer erros, em virtude da imaturidade inerente ao sistema nervoso. Pela frequência de erros encontrados no presente estudo, identifica-se a característica desta amostra: composta por crianças que foram avaliadas por diversos problemas, desde os cognitivos até os emocionais. Tal fato justifica o percentual de erros ser maior na faixa etária de 12 anos, idade em que, segundo Koppitz (1989), já é possível constatar um amadurecimento neurológico, a fim de copiar as figuras do Bender praticamente sem erros.

Concluindo, o Bender, pelo sistema Koppitz, apresenta evidências de validade como instrumento maturacional, com vistas a identificar diferenças na cópia das figuras quanto à idade. Da mesma forma, este estudo também possibilitou a constatação do tipo de erro mais executado pela criança, nesta amostra clínica, assim como a idade em que este erro é mais frequente. Todavia, vale destacar que pesquisas futuras devem ser feitas com o propósito de averiguar resultados característicos de uma população supostamente normal.

\section{Referências}

Arzeno, M. E. (1995). Psicodiagnóstico - novas contribuições clínicas. Porto Alegre, RS: Artes Médicas.

Bender, L. (1955). Test Gestaltico Visomotor (B-G) - uso y aplicaciones clínicas. Buenos Aires: Paidós.

Clawson, A. (1992). Bender Infantil - Manual de diagnóstico clínico ( 7 a $\mathrm{ed}$.). Porto Alegre, RS: ArtMed.

Cunha, J. A. (2000). Bender na criança e no adolescente. Em Cunha, J. A. e cols. Psicodiagnóstico-V (pp. 295-316) (5.ed. revisada e ampliada). Porto Alegre, RS: Artes Médicas.
Kacero, E. (2005). Test Gestaltico Visomotor de Bender: una puesta em espacio de figuras. Buenos Aires: Lugar Editorial.

Kaefer, H. (2006). Semiologia Psicológica. Em Ohlweiler, L., Riesgo, R. S. \& Rotta, T. N. (Orgs.), Transtornos de aprendizagem - abordagem neurobiológica e multidisciplinar (pp. 87-102). Porto Alegre, RS: ArtMed.

Koppitz, E. (1960). The Bender Gestalt Test for Children: a normative study. Journal Clinical Psychology, 16, 432-435.

Koppitz, E. (1989). O Teste Gestaltico Bender para Crianças. Porto Alegre, RS: ArtMed.

Noronha, A. P. P. \& Mattos, R. M. C. B. (2006). Koppitz e Bender-sistema de pontuação gradual: comparação entre sistemas de avaliação. Psicologia Escolar e Educacional, 10, 223-234.

Noronha, A. P. P., Santos, A. A. A. \& Sisto, F. F. (2007). Evidências de Validade do Bender sistema de pontuação gradual (B - SPG). Psicologia: Reflexão e Crítica, 20(2), 335-341.

Ocampo, M. L. S., Arzeno, M. E. G., \& Piccolo, E. G. (1981). O processo psicodiagnóstico e as técnicas projetivas. São Paulo, SP: Martins Fontes.

Ohlweiler, L. \& Guardiola, A. (Orgs.). (2006). Disgnosias. Em Ohlweiler, L., Rotta, N. T. \& Riesgo, R. S. (Orgs.). Transtornos de aprendizagem abordagem neurobiológica e multidisciplinar (pp. 249268). Porto Alegre, RS: ArtMed.

Oliveira, M. S. (1985). O Teste de Bender e o Desenho da Figura Humana como preditores do rendimento escolar (Dissertação de Mestrado). Porto Alegre: PUCRS - Faculdade de Psicologia.

Sisto, F. F., Noronha, A. P. P. \& Santos A. A. A. (2004). Distorção de forma no Teste de Bender: questionando seu critério de validade. Revista da Universidade Federal Fluminense, 3(1), 13-20.

Wechsler, S. M. \& Guzzo, R. S. L. (2005). Avaliação psicológica: perspectiva internacional ( $2^{\text {a }}$ ed.). São Paulo, SP: Casa do Psicólogo.
Recebido em outubro de 2008 Reformulado em abril de 2009 Aprovado em abril de 2009 
Sobre os autores:

Roselaine Berenice Ferreira da Silva é graduada em Psicologia (Universidade de Caxias do Sul, 1991), mestra em Desenvolvimento Regional (Universidade de Santa Cruz do Sul, 2000) e doutora em Psicologia (Pontifícia Universidade Católica do rio Grande do Sul, 2008). Como docente na Universidade de Santa Cruz do Sul, desde 1997, leciona as disciplinas de Técnicas Psicométricas, Técnicas Projetivas, Psicodiagnóstico e Psicopatologia, sendo também coordenadora do curso de Pós-Graduação em Avaliação Psicológica, na mesma instituição.

Cristiane Friedrich Feil é graduanda do curso de Psicologia da PUCRS (ingresso em 2005) e bolsista FAPERGS com os projetos "Evidência de validade do teste Gestáltico Visomotor Bender" (2006 a 2007) e "Atualização das normas de correção do teste Bender" (2007 a 2009).

Maria Lúcia Tiellet Nunes é graduada em Psicologia (Pontifícia Universidade Católica do Rio Grande do Sul, 1974), mestra em Psicologia (Psicologia Social, na Universidade Federal da Paraíba, 1979) e doutora em Psicologia - Tratamento e Prevenção. (Freie Universitat Berlin, FUB, Alemanha, 1989). Atua como docente no Curso de Graduação e Pós-Graduação em Psicologia, na Pontifícia Universidade Católica do Rio Grande do Sul, coordenadora do Programa de Pós-Graduação em Psicologia da mesma instituição e pesquisadora líder do Grupo de Pesquisa Avaliação e Intervenção em Psicoterapia e Psicossomática do programa de Pós-Graduação da PUCRS. 